\title{
Evolution of the Masses of Neutron Stars in Binary Systems
}

\author{
A. I. Bogomazov', M. K. Abubekerov², V. M. Lipunov², and A. M. Cherepashchuk ${ }^{2}$ \\ ${ }^{1}$ Moscow State University, Moscow, Russia \\ ${ }^{2}$ Sternberg Astronomical Institute, Moscow State University, Moscow, Russia \\ Received August 10, 2004; in final form, September 20, 2004
}

\begin{abstract}
We study the growth of the masses of neutron stars in binary systems due to the accumulation of mass from the optical donors accreted onto the neutron-star surface. Possible scenarios for this accretion are considered. The masses and magnetic-field strengths of radio pulsars derived using populationsynthesis methods are compared to the observational data. The population-synthesis analysis indicates that a neutron star can increase its mass from the standard value of $m_{x} \simeq 1.35 M_{\odot}$ to the OppenheimerVolkoff limit, $m_{x} \simeq 2.5 M_{\odot}$, via accretion from a companion. (C) 2005 Pleiades Publishing, Inc.
\end{abstract}

\section{INTRODUCTION}

Data from orbiting $\mathrm{X}$-ray observatories have enabled the discovery of about a thousand X-ray sources in the Milky Way and nearby galaxies [1, 2]. Most are close binary systems in which an optical component supplies mass to a neutron star. Accretion onto the surface of a neutron star at subrelativistic velocities results in an enormous release of X-ray energy and luminosities of the order of $10^{36}-10^{37} \mathrm{erg} / \mathrm{s}$ [3-7]. The mechanism for this powerful generation of energy was first predicted and demonstrated by Zeldovich [3] and Salpeter [4].

According to our current understanding of such systems, superaccretion and hyperaccretion may occur in addition to ordinary accretion, depending on the parameters of the binary and the mass-loss rate of the optical star. In the case of superaccretion, the $\mathrm{X}$-ray luminosity reaches the Eddington limit, thereby limiting further accretion. This happens at accretion rates of $\dot{m} \simeq 10^{-4}-10^{-5} M_{\odot} /$ yr. Chevalier [8] showed that, when the accretion rate is higher $(\dot{m} \simeq$ $10^{-2}-10^{-3} M_{\odot} / \mathrm{yr}$ ), energy is radiated away not by high-energy photons, but by neutrinos. Over the typical time scale for the hyperaccretion stage of $\sim 10^{2}$ yr, up to $\sim 1 M_{\odot}$ of matter may be incident onto the surface of the neutron star.

In recent years, masses have been measured for more then ten radio and X-ray pulsars, and the number of mass estimates for neutron stars is continuously growing. We have carried out a populationsynthesis analysis using the "Scenario Machine" [9] with the aim of elucidating possible values of the masses and magnetic-field strengths of neutron stars in binary systems.

\section{THREE REGIMES OF MASS ACCRETION BY NEUTRON STARS}

A considerable fraction of observed neutron stars have increased their masses in the course of their evolution, or are still increasing their masses (e.g., in $\mathrm{X}$-ray sources). But how large can this mass increase be? It is clear that the only origin of a mass increase is accretion. It is evident that the overall change in the mass of a neutron star is determined not only by the accretion rate, but also by the duration of the accretion stage:

$$
\Delta M=\int_{0}^{T_{a}} \dot{M} d t=\dot{M} T_{a},
$$

, where $\dot{M}$ is the mean accretion rate and $T_{a}$ is the lifetime of the accretion stage. We emphasize that, in the case under consideration, the accretion rate is the amount of matter falling onto the surface of the neutron star per unit time, and can differ significantly from the values indicated by the classical BondiHoyle formulas. Three regimes of accretion are possible in a close binary containing a neutron star: ordinary accretion, superaccretion, and hyperaccretion.

\section{Accretion}

An ordinary accretion regime is realized when all the matter captured by the gravitational field of the neutron star falls onto its surface. This is possible only if the radiation pressure and electromagnetic forces associated with the magnetic field of the star and its rotation are small compared to the gravitational force.

In this case, the increase in the mass will be precisely determined by the gas dynamics of the accretion at the gravitational-capture radius or, if the donor 
Table 1. Masses and rotational periods of neutron stars in radio pulsar + neutron star systems

\begin{tabular}{l|c|c|c|c|c}
\hline \multicolumn{1}{c|}{ System } & $p_{\text {rot }}, \mathrm{ms}$ & $\dot{p}_{\text {rot }}, \mathrm{s} / \mathrm{s}$ & $B, \mathrm{G}$ & $m_{\mathrm{PSR}}, M_{\odot}$ & References \\
\hline $\mathrm{J} 1518+4904$ & 40.9 & $4.0 \times 10^{-20}$ & $2.6 \times 10^{9}$ & $1.56_{-1.20}^{+0.20}$ & {$[13,14]$} \\
$\mathrm{B} 1534+12$ & 37.9 & $2.4 \times 10^{-18}$ & $1.9 \times 10^{10}$ & $1.3332 \pm 0.0020$ & {$[13,15,16]$} \\
$\mathrm{B} 1913+16$ & 59.0 & $8.6 \times 10^{-18}$ & $4.6 \times 10^{10}$ & $1.4408 \pm 0.0006$ & {$[13,17,18]$} \\
$\mathrm{B} 2127+11 \mathrm{C}$ & 30.5 & $1.0 \times 10^{-17}$ & $3.5 \times 10^{10}$ & $1.349 \pm 0.080$ & {$[13,19]$} \\
$\mathrm{J} 0737-3039(1)$ & 22.7 & $1.7 \times 10^{-18}$ & $1.3 \times 10^{10}$ & $1.337 \pm 0.010$ & {$[20]$} \\
$\mathrm{J} 0737-3039(2)$ & 2773.5 & $8.8 \times 10^{-16}$ & $3.2 \times 10^{12}$ & $1.250 \pm 0.010$ & {$[20]$} \\
\hline
\end{tabular}

fills its Roche lobe, by the component-mass ratio and the evolutionary status of the optical component. In this case, the accretor is observed as an X-ray source with luminosity

$$
L_{x}=\dot{M} \frac{G M_{x}}{R_{*}},
$$

where $M_{x}$ and $R_{*}$ are the mass and radius of the neutron star. The accretion rate $\dot{M}$ is determined by the Bondi-Hoyle formula

$$
\dot{M}=\pi R_{G}^{2} \rho v,
$$

where $R_{G}$ is the gravitational-capture radius of the neutron star, $v$ the velocity of the gas flow relative to the neutron star, and $\rho$ the density of the gas.

The X-ray luminosity of the accretor $L_{x}$ and its other main parameters can be used to estimate the mass $\Delta M$ accumulated during the accretion phase:

$$
\Delta M=\frac{L_{x} R_{*} T_{a}}{G M_{x}} .
$$

For massive stars, with lifetimes of less than $10^{7} \mathrm{yr}$, accretion via the stellar wind of the donor is negligibly small at the level of accuracy provided by modern computations. However, the mass increase for low-mass systems containing one or more components that fill their Roche lobes can be considerable, reaching several solar masses. We shall show this below.

\section{Superaccretion}

The most thorough analysis of the superaccretion regime for a magnetized neutron star was carried out by Lipunov [10]. Despite the absence of detailed models for supercritical disk accretion (supercritical accretion is realized precisely via an accretion disk), it is possible to estimate the main characteristics of the process - the accretion rate, magnetosphere radius, and evolution equations. Accretion is considered to be supercritical when the energy released at the radius where the accretion flow stops exceeds the Eddington limit:

$$
\begin{gathered}
\dot{M} \frac{G M_{x}}{R_{\text {stop }}}>L_{E d d}=1.38 \times 10^{38}\left(M_{x} / M_{\odot}\right) \mathrm{erg} / \mathrm{s}, \\
\dot{M}>\dot{M}_{\text {crit }}=10^{-8} M_{\odot} / \mathrm{yr},
\end{gathered}
$$

where $R_{\text {stop }}$ is either the radius of the neutron star or the magnetosphere radius $R_{A}$.

For strongly magnetized neutron stars with magnetic fields $B \gg 10^{8} \mathrm{G}$, all matter arriving from the magnetosphere is accreted onto the magnetic poles, where the corresponding gravitational energy is released. If the blackbody temperature $T$, roughly estimated as

$$
S \sigma T^{4}=\dot{M} \frac{G M_{x}}{R_{*}},
$$

is higher than $5 \times 10^{9} \mathrm{~K}(S$ is the area of the base of the accretion column), most of the energy will escape the surface from the neutron star in the form of neutrinos, and, hence, will not hinder accretion [11, 12]. In this case, the rate at which the neutron star accumulates mass will be

$$
\dot{M} \simeq \dot{M}_{\text {crit }}\left(\frac{R_{A}}{R_{*}}\right)^{2} \gg \dot{M}_{\text {crit }}
$$

If the temperature becomes lower, there will be an upper limit on the accretion rate equal to the standard Eddington limit.

\section{Hyperaccretion}

A considerable fraction of neutron stars in binary systems pass through a common-envelope stage in the course of their evolution. In this case, the neutron star is effectively immersed in its companion, and for a short time $\left(10^{2}-10^{4} \mathrm{yr}\right)$ moves along spirals inside a region of dense matter. The formal accretion rate estimated using the Bondi-Hoyle formulas is four to six orders of magnitude higher than the critical rate and, as was suggested by Chevalier [8], this may result in 
Table 2. Masses and rotational periods of neutron stars in radio pulsar+white dwarf (optical star) systems

\begin{tabular}{c|c|c|c|c|c}
\hline System & $p_{\text {rot }}, \mathrm{ms}$ & $\dot{p}_{\text {rot }}, \mathrm{s} / \mathrm{s}$ & $B, \mathrm{G}$ & $m_{\mathrm{PSR}}, M_{\odot}$ & References \\
\hline $\mathrm{J} 0437-4715$ & 5.76 & $1.0 \times 10^{-20}$ & $4.86 \times 10^{8}$ & $1.58 \pm 0.18$ & {$[13,21]$} \\
$\mathrm{J} 0621+1002$ & 28.9 & $4.70 \times 10^{-20}$ & $2.36 \times 10^{9}$ & $1.70_{-0.63}^{+0.59}$ & {$[22,23]$} \\
$\mathrm{J} 1012+5307$ & 5.26 & $1.20 \times 10^{-20}$ & $5.09 \times 10^{8}$ & $1.7 \pm 1.0$ & {$[13]$} \\
$\mathrm{J} 1141-6545$ & 394 & $4.29 \times 10^{-15}$ & $2.63 \times 10^{12}$ & $1.30 \pm 0.02$ & {$[24,25]$} \\
$\mathrm{J} 1713+0747$ & 4.57 & $8.52 \times 10^{-21}$ & $4.00 \times 10^{8}$ & $1.65 \pm 0.45$ & {$[13,26,27]$} \\
$\mathrm{B} 1802-07$ & 23.1 & $4.70 \times 10^{-19}$ & $6.67 \times 10^{9}$ & $1.26_{-0.67}^{+0.15}$ & {$[13]$} \\
$\mathrm{B} 1855+09$ & 5.36 & $1.78 \times 10^{-20}$ & $6.26 \times 10^{8}$ & $1.57_{-0.20}^{+0.25}$ & {$[13,27]$} \\
$\mathrm{B} 2303+46$ & 1066.4 & $5.6 \times 10^{-16}$ & $1.57 \times 10^{12}$ & $1.34 \pm 0.10$ & {$[13,28]$} \\
$\mathrm{J} 1740-5340$ & 3.65 & $1.6 \times 10^{-19}$ & $1.55 \times 10^{9}$ & $1.53 \pm 0.19$ & {$[29-31]$} \\
\hline
\end{tabular}

hyperaccretion, when all the energy is carried away by neutrinos for the reasons described above. There are currently no detailed theories for hyperaccretion or the common-envelope stage. The amount of matter accreted by the neutron star can be estimated as

$$
\begin{aligned}
& \Delta M=\int_{0}^{T_{\text {hyper }}} \frac{1}{4}\left(\frac{R_{G}}{a}\right)^{2} \dot{M} d t \\
& \simeq \frac{1}{4}\left(M_{\text {opt }}-M_{\text {core }}\right)\left(\frac{M_{x}}{M_{\text {opt }}}\right)^{2},
\end{aligned}
$$

where $T_{\text {hyper }}$ is the duration of the hyperaccretion stage, $R_{G}$ the gravitational-capture radius of the radio pulsar, $a$ the initial semimajor axis of the close binary orbit, $M_{\text {core }}$ the mass of the core of the optical star, and $M_{o p t}$ and $M_{x}$ are the total masses of the optical star and of the radio pulsar at the onset of the hyperaccretion stage.

Inserting the parameters of observed X-ray systems into (3), (4), and (6), we obtain for the masses of matter accreted in various accretion processes values from $\sim 0.1 M_{\odot}$ to $\sim 1 M_{\odot}$.

In recent years, masses have been measured for more than ten neutron stars, on average, with accuracies of $\sim(0.3-0.5) M_{\odot}$. Thus, the observational data already allow empirical tests of the hypothesis that neutron stars in binary systems can accumulate mass.

\section{OBSERVED MASSES AND MAGNETIC FIELDS OF NEUTRON STARS}

We consider here binary systems in which the mass-transfer stage is already over-radio pulsars paired with neutron stars and white dwarfs. The masses of the radio pulsars and other parameters of these systems are listed in Tables 1 and 2 (see also Fig. 2 below). The masses listed in the tables are dynamical estimates derived from radial-velocity curves for point-mass models. Most of the values are taken from the 1999 review of Thorsett and Chakrabarty [13]. The uncertainties in the mass estimates for radio pulsars paired with neutron stars (Table 1) are given for the $68 \%$ confidence level. The uncertainties in the mass estimates for radio pulsars paired with white dwarfs (Table 2 ) are given for the $95 \%$ confidence level.

The observed rotation of an accreting neutron star is the result of two competing processes: acceleration due to the transfer of angular momentum from inner regions of the accretion disk, and braking due to the interaction of the toroidal component of the neutron star's magnetic field with outer regions of the accretion disk [10]. An equilibrium between the accelerating and braking torques is established over a time

$$
t_{e q} \simeq \frac{I \omega}{\dot{M} \sqrt{G M R_{c}}} \simeq \frac{M_{x}}{\dot{M}}\left(\frac{R_{x}}{R_{c}}\right)^{3} \ll \frac{M_{x}}{\dot{M}} .
$$

The equilibrium rotational period of a neutron star $p_{e q}$ is proportional to its magnetic dipole momentum $\mu$ (see, e.g., [10]). Thus, a neutron star that has experienced an accretion stage should display a correlation between its magnetic field and rotation. We accordingly investigated for the radio pulsars considered here the dependence between their observed rotational periods $p_{\text {rot }}$ and the rates at which their rotations are slowing $\dot{p}_{\text {rot }}$ (Fig. 1 ). The value of $\dot{p}_{\text {rot }}$ is proportional to the square of the magnetic dipole momentum of the radio pulsar $\mu$ [see (7)]. Figure 1 shows that the radio pulsars considered by us display a clear correlation between $\dot{p}_{\text {rot }}$ and $p_{\text {rot }}$, suggesting that the radio pulsars listed in Tables 1 and 2 are neutron stars that have experienced an accretion stage. 
Table 3. Masses and rotational periods of neutron stars in close $\mathrm{X}$-ray binaries

\begin{tabular}{l|c|c|c}
\hline \multicolumn{1}{c|}{ System } & $p_{\text {rot }}, \mathrm{s}$ & $m_{N S}, M_{\odot}$ & References \\
\hline 2A 1822-371 & 0.593 & $0.97 \pm 0.24$ & {$[33,34]$} \\
Her X-1 & 1.24 & $1.50 \pm 0.30$ & {$[35,36]$} \\
Cen X-3 & 4.8 & $1.22_{-0.14}^{+0.15}$ & {$[37,38]$} \\
LMC X-4 & 13.5 & $1.63_{-0.47}^{+0.42}$ & {$[37,39,40]$} \\
SMC X-1 & 0.71 & $1.48_{-0.42}^{+0.47}$ & {$[37,41]$} \\
4U 1538-52 & 528.2 & $1.18_{-0.27}^{+0.29}$ & {$[37,42]$} \\
Vela X-1 & 283 & $1.93_{-0.21}^{+0.19}$ & {$[37,43]$} \\
\hline
\end{tabular}

The magnetic fields of radio pulsars can be derived assuming the neutron star loses angular momentum via the magneto-dipole mechanism. The strength of the magnetic field $B$ is computed as

$$
\begin{gathered}
\mu=\left(\frac{3 I c^{3} p_{\text {rot }} \dot{p}_{r o t}}{8 \pi}\right)^{1 / 2}, \\
B=2 \mu / R_{*}^{3},
\end{gathered}
$$

where $p_{\text {rot }}$ is the rotational period of the radio pulsar, $\dot{p}_{\text {rot }}$ the rate of deceleration of the rotation, $c$ the speed of light, $I$ the radio pulsar's moment of inertia, which is taken to be $10^{45} \mathrm{~g} \mathrm{~cm}^{2}, \mu$ its magnetic-dipole moment, and $R_{*}$ its radius, which we take to be $10^{6} \mathrm{~cm}$.

It is impossible to study accretion without mentioning $\mathrm{X}$-ray pulsars. The magnetic fields $B$ of all known X-ray pulsars derived from both gyroscopic lines in their X-ray spectra [32] and their X-ray luminosities [10] exceed $10^{12} \mathrm{G}$. The masses of $\mathrm{X}$-ray pulsars are listed in Table 3; the errors are given for the $95 \%$ confidence level. However, we did not include $\mathrm{X}$-ray pulsars in our study for two reasons. First, the accretion onto the neutron stars in these systems has just started. Most X-ray pulsars are accreting mass from the stellar wind of the optical companion (the only exception known with certainty is the $\mathrm{X}$-ray pulsar Her $\mathrm{X}-1$, which is accreting matter from a disk). Hence, X-ray pulsars have not had time to considerably increase their masses (by one to several tenths of a solar mass). Second, mass estimates for $\mathrm{X}$-ray pulsars remain fairly inaccurate. For example, the high mass of the neutron star in the Vela X-1 system $\left(m_{x}=1.93 M_{\odot}\right)$ derived from a best-fit model is not trustworthy, since the model does not adequately describe the observational data (for more details, see [37]). For these reasons, we did not consider $\mathrm{X}$-ray pulsars in this study.

We assumed that the magnetic fields of neutron stars decay exponentially to some minimum value
$B_{\min }=8 \times 10^{7} \mathrm{G}$ and do not decay further:

$$
\left\{\begin{array}{l}
B_{0} \exp \left(-t / t_{d}\right), \quad t<t_{d} \ln \left(B_{0} / B_{\text {min }}\right), \\
8 \times 10^{7}, \quad t \geq t_{d} \ln \left(B_{0} / B_{\text {min }}\right) .
\end{array}\right.
$$

The parameters $B_{0}$ and $t_{d}$ in (9) are the initial field strength and the time scale for decay of the field. $\mathrm{Tt}$ is not possible to determine the exact ages of neutron stars since $B_{0}$ and $t_{d}$ are not known. However, the magnetic-field strength itself can serve as an indicator of age. For this reason, we constructed the dependence between the magnetic-field strength and the neutron-star mass, shown in Figs. 2 and 3. We will call this dependence the magnetic field-mass diagram.

Figure 2 shows the observational estimates of the masses of radio pulsars together with their uncertainties. The errors are fairly large, and mask the scatter of the central values of observed masses. For this reason, we have plotted the central values only in Fig. 3.

The central values of the radio-pulsar masses show certain systematic variations (Fig. 3). We can distinguish two possible branches in the magnetic field-mass diagram: one showing evolution of neutron stars without increasing mass (the vertical gray strip in Fig. 3), and the other showing evolution with increasing mass (slanted gray strip in Fig. 3).

These observational data (despite their large errors) require interpretation. For this purpose, we carried out a population-synthesis analysis using the "Scenario Machine" [9]. This enabled us to investigate the range of possible observed parameters of the neutron stars, and also to determine qualitatively the types of evolutionary tracks that result in increases in the mass of a neutron star.

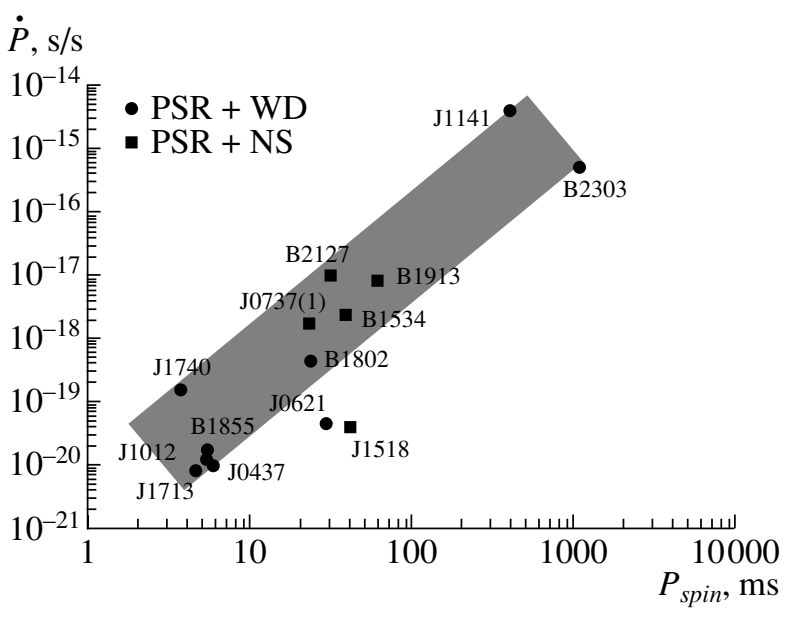

Fig. 1. Dependence between deceleration of the rotation $\dot{p}_{\text {rot }}$ and the rotational period $p_{\text {rot }}$ for radio pulsars in pairs with white dwarfs (filled circles) and with neutron stars (filled squares). 


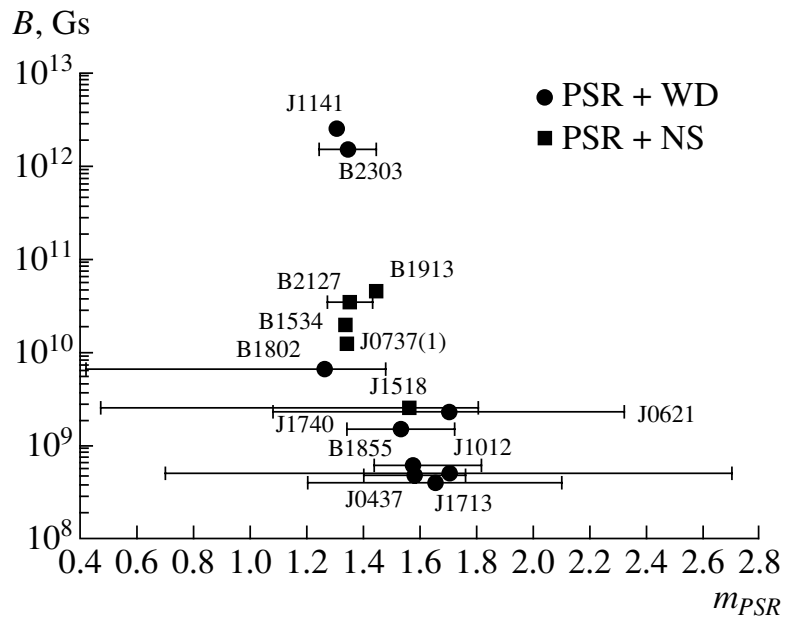

Fig. 2. Relation between the masses $m_{\mathrm{PSR}}$ and magneticfield strengths $B$ of radio pulsars (errors in $m_{\mathrm{PSR}}$ are shown). The filled squares and circles correspond to radio pulsars in pairs with neutron stars (PSR + NS) and white dwarfs (PSR+WD).

\section{POPULATION SYNTHESIS}

We used the "Scenario Machine" to carry out population syntheses for 19.5 million binary systems [9]. The initial component masses $M_{1}$ and $M_{2}$ were varied between $5 M_{\odot}$ and $120 M_{\odot}$, assuming a flat distribution of initial component-mass ratios. The initial semimajor axis of the orbit could have any value in the range $\left(10-10^{6}\right) R_{\odot}$.

Based on the population-synthesis output, we identified systems that resulted in the formation of radio pulsar+neutron star (PSR+NS) and radio pulsar+white dwarf (PSR+WD) pairs. We focused on radio pulsars in systems with degenerate dwarfs for our study.

Among the many parameters of binaries with radio pulsars (PSR $+\mathrm{NS}$ and $\mathrm{PSR}+\mathrm{WD}$ ), we were interested in the magnetic-field strengths $B$ and masses $m_{\mathrm{PSR}}$ of the radio pulsars. Most radio pulsars in pairs with degenerate companions inevitably passed through an accretion phase, thereby increasing their masses by some amount $\Delta M$ (see Eq. (1)). The masses and magnetic-field strengths for the PSR+NS systems are presented for the time when the neutron star formed, while the masses and magnetic-field strengths for the PSR+WD systems are presented for the time of formation of the white dwarf. In other words, the magnetic fields and masses correspond to the "birth points" (on the evolutionary tracks) of the PSR+NS and PSR+WD binaries.

The evolution of radio pulsar+optical star systems depends strongly on the magnetic-field strength $B$ and its decay time $t_{d}$. Since this decay time is not

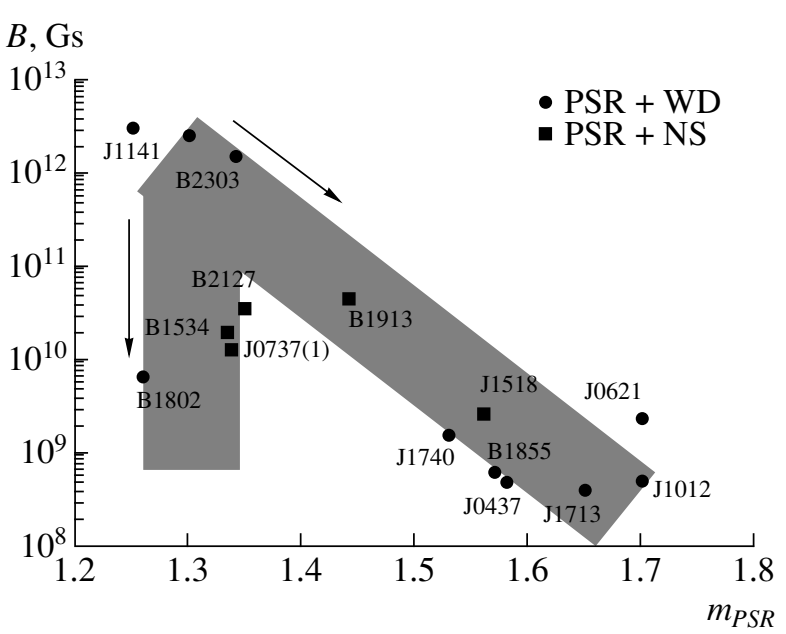

Fig. 3. Relation between $m_{\mathrm{PSR}}$ and $B$ for radio pulsars (the errors for $m$ are not shown) with neutron-star (PSR+NS; filled squares) and white-dwarf (PSR+WD; filled circles) companions. The arrows show the directions of the evolution of the mass and magnetic-field strength of the radio pulsars.

known, we carried out population-synthesis computations for several magnetic-field decay times: $t_{d}=$ $10^{7}, 5 \times 10^{7}$, and $10^{8}$ yr. The modeling was carried out for regimes both with and without hyperaccretion. It was assumed that all neutron stars have initial masses $1.35 M_{\odot}$ and initial magnetic fields $2 \times$ $10^{12} \mathrm{G}$. Recall that we assumed that the decay of the neutron-star magnetic field was exponential, $B=$ $B_{0} \exp \left(-t / t_{d}\right)$ [see (9)].

The population-synthesis algorithm also assumed that the anisotropic kick velocities acquired by the neutron stars during the supernova explosions in which they are formed have a Maxwellian-like distribution with dispersion $v_{0}=180 \mathrm{~km} / \mathrm{s}$ [44], and that the spatial distribution of the kick velocities is isotropic. The Chandrasekhar mass was set equal to $m_{C h}=1.4 M_{\odot}$. The Oppenheimer-Volkoff limit was taken to be $m_{\mathrm{OV}}=2.5 M_{\odot}$.

Figures $4-8$ show the magnetic fields of the radio pulsars and their masses at the birth epochs of the PSR+NS and PSR+WD pairs derived from the population synthesis. Let us now consider in detail each possible scenario for the evolution of the masses and magnetic fields of the radio pulsars in these systems.

\section{Radio Pulsars in PSR+NS Binaries (Population Synthesis with Hyperaccretion)}

The population synthesis for the 19.5 million initial systems resulted in the formation of $\sim 7 \times 10^{4}$ PSR+NS systems. PSR+NS systems are produced by the evolution of binaries with initial componentmass ratios $q=M_{1} / M_{2}=1-4$, initial component 


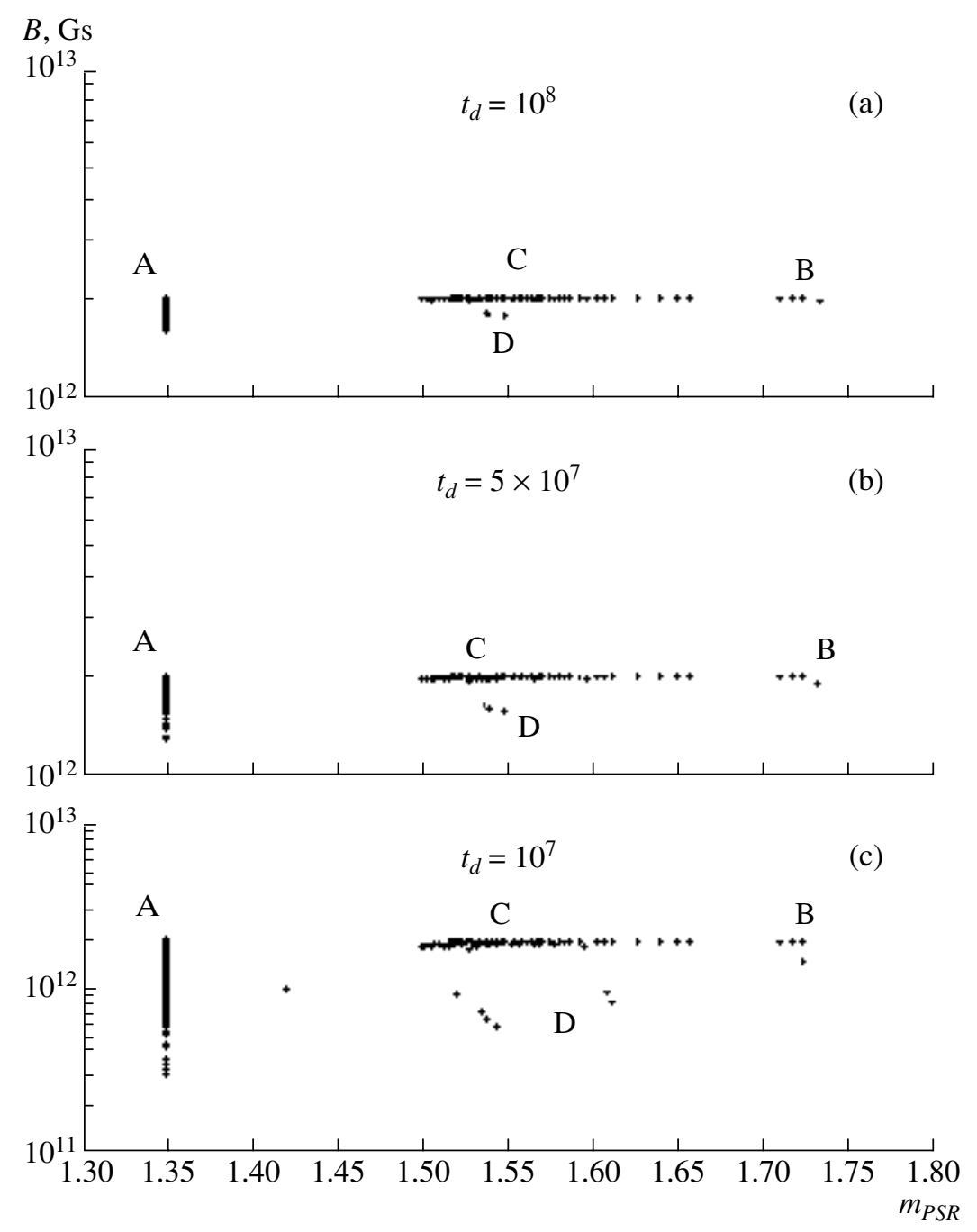

Fig. 4. Masses and magnetic-field strengths for radio pulsars in the $\sim 7 \times 10^{4} \mathrm{PSR}+\mathrm{NS}$ binaries generated in the population synthesis for 19.5 million initial systems taking into account hyperaccretion. A detailed description of selected groups of pulsars is given in the text.

masses $M_{1} \geq 10 M_{\odot}, M_{2} \geq 10 M_{\odot}$, and initial semimajor orbital axes $a \simeq\left(10-10^{3}\right) R_{\odot}$.

Figure 4 shows the magnetic fields and masses of radio pulsars in binaries with neutron stars at the epoch when the PSR+NS system was born. Figures $4 \mathrm{a}-\mathrm{c}$ present the population-synthesis results for magnetic-field decay time scales of $t_{d}=10^{8}, 5 \times$ $10^{7}$, and $10^{7} \mathrm{yr}$, respectively. Four groups of radio pulsars can be distinguished in all the plots. The four groups of qualitatively similar (in terms of their accretion) groups of pulsars are for convenience labelled A, $\mathrm{B}, \mathrm{C}$, and $\mathrm{D}$.

The radio pulsars of group A (Figs. $4 a-4 c$ ) do not experience accretion, superaccretion, or hyperaccretion, so that their masses do not change: $m_{\mathrm{PSR}}=$ $1.35 M_{\odot}$. However, their magnetic-field strengths decrease. These radio pulsars are formed from binaries with initial component masses $M_{1} \simeq 14-40 M_{\odot}$,
$M_{2} \simeq 11-15 M_{\odot}$, and initial semimajor orbital axes $a \simeq 10^{2}-10^{3} R_{\odot}$.

The radio pulsars of group $\mathrm{B}$ form during the evolution of binaries with $M_{1} \simeq 15-22 M_{\odot}, M_{2} \simeq$ $14-19 M_{\odot}$, and $a \simeq 10-10^{3} R_{\odot}$. These radio pulsars accumulate mass during superaccretion $(\Delta M \simeq$ $\left.0.1-0.2 M_{\odot}\right)$ and hyperaccretion $(\Delta M \simeq$ $\left.0.2-0.3 M_{\odot}\right)$ stages. We can see that the mass is accumulated relatively rapidly $\left(t<t_{d}\right)$, and the magnetic-field strength does not have time to change significantly $\left(B \simeq 2 \times 10^{12} \mathrm{G}\right.$ ).

The radio pulsars of group $C$ are produced by binary systems whose components have comparable masses $(q \simeq 1)$, with the individual component masses being $M_{1} \simeq 30-40 M_{\odot}, M_{2} \simeq 30-35 M_{\odot}$. The initial semimajor orbital axes are between $\sim 5 \times$ $10 R_{\odot}$ and $\sim 5 \times 10^{2} R_{\odot}$. These radio pulsars pass 


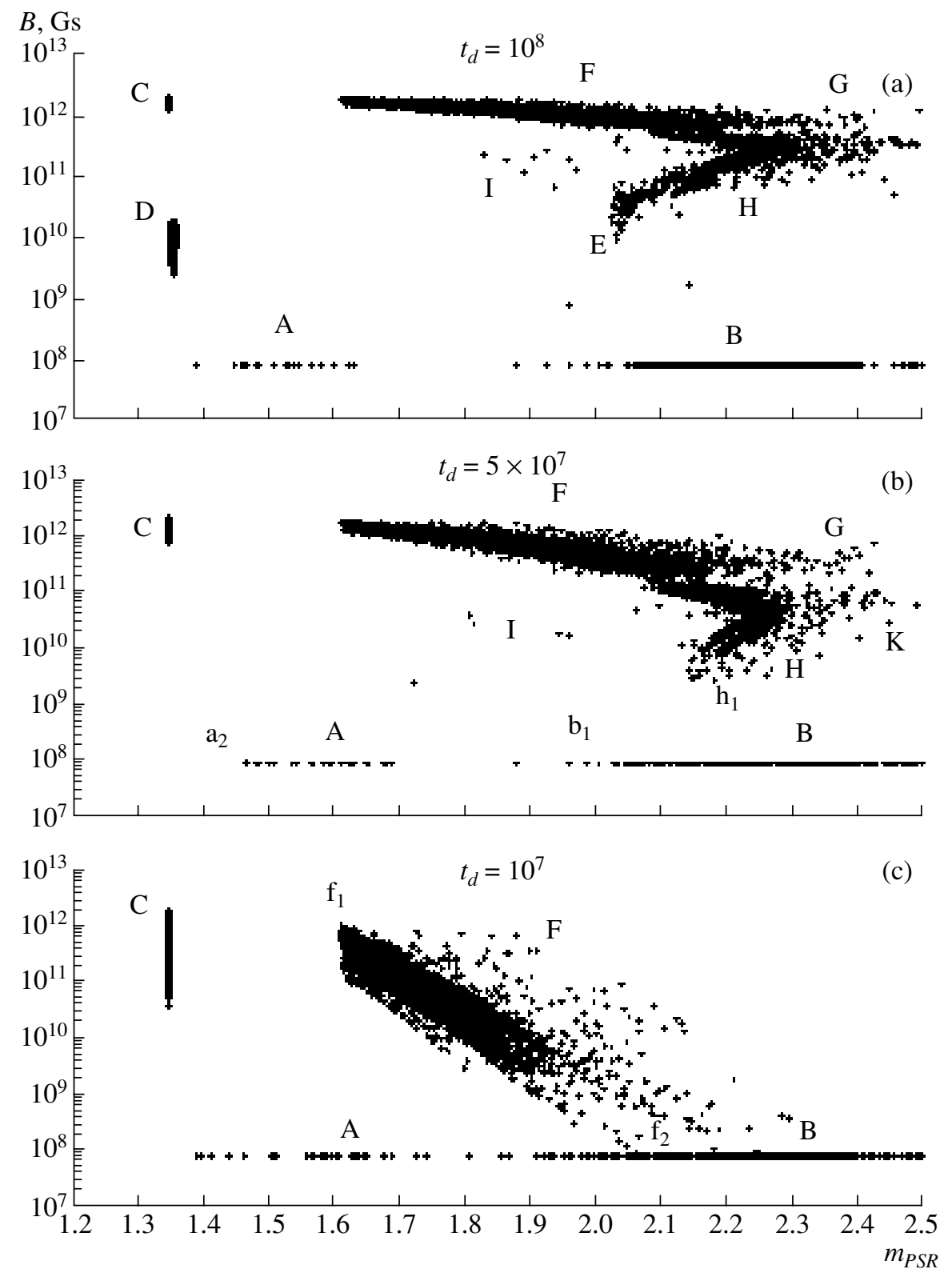

Fig. 5. Same as Fig. 4 for PSR + WD systems $\left(\sim 16 \times 10^{4}\right.$ objects $)$.

through a hyperaccretion stage only, and increase their masses exclusively during this stage $(\Delta M \simeq$ $\left.0.15-0.20 M_{\odot}\right)$. The magnetic-field strength does not have time to change significantly ( $B \simeq 2 \times 10^{12} \mathrm{G}$ ); i.e., the mass is accumulated on a time scale $t<t_{d}$.

Like the group-B pulsars, the radio pulsars of group D experience both superaccretion and hyperaccretion. However, in contrast to the group-B pulsars, they accumulate only a small amount of matter in the superaccretion stage. For instance, the pulsar masses increase by no more than $0.03 M_{\odot}$ in the superaccretion stage, while $\Delta M \simeq 0.3 M_{\odot}$ in the hyperaccretion stage. The initial component masses for this group are $M_{1}=13-52 M_{\odot}, M_{2}=10-20 M_{\odot}$, and the initial semimajor orbital axes are $a \simeq(2-9) \times 10^{2} R_{\odot}$.

The locations of pulsars in groups A, B, C, and D in Figs. $4 a-4 c$ are similar. The groups differ only in their magnetic-field strengths, due to the effect of different field-decay time scales. For example, in Fig. 4c, which plots objects with the shortest decay times $\left(t_{d}=10^{7} \mathrm{yr}\right)$, the radio pulsars are located lower than their analogs in Figs. 4a and 4b. These differences in $t_{d}$ do not lead to qualitative differences in the evolutionary scenarios for the formation of PSR+NS systems.

The population-synthesis results show that a radio pulsar can increase its mass from $M \simeq 1.35 M_{\odot}$ to 
$M \simeq 1.70 M_{\odot}$ during the superaccretion and hyperaccretion stages, over a time $t \leq t_{d}$.

\section{Radio Pulsars in PSR+WD Binaries (Population Synthesis with Hyperaccretion)}

The population synthesis for 19.5 million initial binaries resulted in the formation of $\sim 16 \times 10^{4}$ PSR+WD binaries. Figure 5 shows the masses and magnetic fields of these pairs at the epoch when the pair is born; i.e., when the white dwarf is formed. As in the previous case, we carried out population syntheses for magnetic-field decay times $t_{d}=10^{8}$, $5 \times 10^{7}$, and $10^{7}$ yr (Figs. 5a-5c).

In contrast to the previous case, variation of the magnetic-field decay time scale $t_{d}$ led to both quantitative and qualitative differences. As $t_{d}$ is decreased, the number of radio pulsars with low magnetic fields grows. This shows up as a change in the slope of the strip formed by the radio pulsars in Fig. 5. Decreasing $t_{d}$ also results in an increase in the number of radio pulsars with the minimum possible magnetic field (Figs. 5a and 5c). The presence of quantitative differences is manifest in the absence of unique connections between the groups of pulsars in Figs. 5a, $5 \mathrm{~b}$, and $5 \mathrm{c}$. This provides evidence that the scenario for variations of the radio-pulsar mass realized in a PSR+WD pair depends on the magnetic-field decay time scale. Note that qualitative changes in the massaccumulation scenario with variation of $t_{d}$ did not occur for the radio pulsars in PSR+NS systems: the groups of radio pulsars in Figs. $4 \mathrm{a}, 4 \mathrm{~b}$, and $4 \mathrm{c}$ are similar.

Let us discuss in detail all the groups of pulsars in Figs. $5 a-5 c$ and the evolutionary tracks resulting in their formation.

\section{Magnetic-field Decay Time Scale $10^{8}$ yr}

Radio pulsars of groups A and B (Fig. 5a) are produced during the evolution of close binaries with initial component masses $M_{1} \geq 10 M_{\odot}$ and $M_{2} \simeq$ $1.1-1.5 M_{\odot} \quad\left(\right.$ group A) $\quad$ or $\quad M_{2} \simeq 1.5-2.5 M_{\odot}$ (group B); the initial semimajor orbital axes are $a=(6-7) \times 10^{2} R_{\odot}$. These pulsars accumulate mass only in an accretion stage. Matter flows toward the pulsars from their low-mass donors through the inner Lagrangian point. The pulsar magnetic field has already decayed to its minimum value by the onset of accretion (the low-mass optical companion fills its Roche lobe on the nuclear time scale, $t \sim$ $10^{8}-10^{9} \mathrm{yr}$ ), and so does not impede the infall of matter onto the surface of the radio pulsar. The pulsars of groups $\mathrm{A}$ and $\mathrm{B}$ are able to increase their masses by $\Delta M \simeq 0.02-1.2 M_{\odot}$ via accretion (Fig. 5a).
Radio pulsars of group C (Fig. 5a) are produced by binaries with $M_{1} \simeq 11-15 M_{\odot}, M_{2} \simeq 5.5-9.5 M_{\odot}$ and $a=2 \times 10^{2}-10^{3} R_{\odot}$. Since the two component masses are comparable $(q \simeq 1.5-2)$ and large, the pulsar magnetic field has not decayed by the time when the white dwarf forms. These pulsars do not pass through accretion, superaccretion, or hyperaccretion stages. The strong pulsar magnetic field, $B \simeq$ $10^{12} \mathrm{G}$, prevents the infall of matter from the donor, with the result that the mass of the radio pulsar remains constant: $m_{\mathrm{PSR}}=1.35 M_{\odot}$ (Fig. 5a).

Radio pulsars of group D (Fig. 5a) are produced by binaries with $M_{1} \simeq 11-16 M_{\odot}, M_{2} \simeq 1.5-4 M_{\odot}$, $q=4-6$, and $a=10^{3}-2 \times 10^{3} R_{\odot}$. The time scale for the nuclear evolution of the primary is two orders of magnitude (a factor of $\sim 10^{2}$ ) shorter than the nuclear time scale of the secondary. Therefore, the magnetic field of the radio pulsar has already decayed to $\sim 10^{10} \mathrm{G}$ by the onset of the accretion stage. The system goes through accretion and superaccretion stages, but, because the field of the neutron star is still strong $\left(\sim 10^{10} \mathrm{G}\right)$, only a few hundredths of a solar mass fall onto the surface of the neutron star $\left(\Delta M \sim 0.01-0.02 M_{\odot}\right)$.

Radio pulsars of group F (Fig. 5a) are produced by binaries with $M_{1} \simeq 10-30 M_{\odot}, M_{2} \simeq 5-8 M_{\odot}$, and $a=(1-3) \times 10^{2} R_{\odot}$. The masses of these pulsars are not increased significantly via superaccretion, $\Delta M \sim$ $0.01 M_{\odot}$, and accumulate the most mass in a hyperaccretion stage: $\Delta M \simeq 0.3-0.7 M_{\odot}$. The $\Delta M$ for the hyperaccretion stage increases with decreasing mass of the optical companion. For example, a radio pulsar with a companion with $M_{2} \simeq 9 M_{\odot}$ accumulates $\Delta M \simeq 0.3 M_{\odot}$ in the hyperaccretion stage, while a radio pulsar with a companion with $M_{2} \simeq 6 M_{\odot}$ accumulates $\Delta M \simeq 0.7 M_{\odot}$ in this stage.

Radio pulsars of group $\mathrm{G}$ (Fig. $5 \mathrm{a}$ ) are produced by binaries with $M_{1} \simeq 13-15 M_{\odot}, M_{2} \simeq 5.5-6.5 M_{\odot}$, and $a=(5-9) \times 10^{2} R_{\odot}$. These pulsars accumulate mass in accretion $\left(\Delta M \simeq 0.2 M_{\odot}\right)$ and hyperaccretion $\left(\Delta M \simeq 0.8 M_{\odot}\right)$ stages.

Radio pulsars of group E (Fig. 5a) are produced by binaries with $M_{1} \simeq 10-15 M_{\odot}, M_{2} \simeq 2.5-4 M_{\odot}$ and semimajor orbital axes from $a=2 \times 10^{2} R_{\odot}$ to $2 \times 10^{3} R_{\odot}$. These pulsars increase their masses consecutively in an accretion, superaccretion, and second accretion stage. The pulsar accumulates a few hundredths of a solar mass in the superaccretion stage. The main mass accumulation occurs in the accretion stages: $\Delta M \simeq 0.7 M_{\odot}$. Like the group $\mathrm{D}$ pulsars, the strength of the magnetic field at the onset of the first accretion stage is $\sim 10^{10} \mathrm{G}$, but, in contrast to the group D pulsars, the group E radio pulsars have component separations an order of magnitude 
smaller. This enables them to increase their masses by $\Delta M \simeq 0.7 M_{\odot}$, rather than $\Delta M \simeq 0.01-0.02 M_{\odot}$.

Radio pulsars of group H (Fig. 5a) pass through a hyperaccretion stage only, in which their masses increase by $\Delta M \simeq 0.8 M_{\odot}$. The initial parameters of their binaries are $M_{1} \simeq 12-26 M_{\odot}, M_{2} \simeq 3-4 M_{\odot}$, and $a \simeq(1-6) \times 10^{2} R_{\odot}$.

The last group of radio pulsars (I) are produced by binaries with $M_{1} \simeq 10-11 M_{\odot}, M_{2} \simeq 3-4 M_{\odot}$, and $a \sim 3 \times 10^{2} R_{\odot}$. They increase their masses only slightly in a superaccretion stage: $\Delta M \simeq 0.02 M_{\odot}$. The pulsar masses grow mainly due to ordinary accretion of matter from the donor through the inner Lagrangian point, with $\Delta M \simeq 0.3-0.4 M_{\odot}$.

\section{Magnetic-field Decay Time Scale $5 \times 10^{7} \mathrm{yr}$}

As was noted above, if the magnetic-field decay time scale $t_{d}$ changes [see Eq. (9)], the evolution of close binaries giving rise to PSR+WD systems changes both quantitatively and qualitatively. Some groups of radio pulsars visible in Fig. 5a disappear, while new groups appear (Fig. 5b). For groups of pulsars displaying only quantitative changes with changes in $t_{d}$, we keep the same notation, while introducing new notation for qualitatively new groups. Let us start with a description of the new groups and the groups that are no longer present.

Groups of radio pulsars D and E in Fig. 5a disappear (Fig. 5b). Thus, the evolutionary scenarios that result in the formation of these groups are not realized when $t_{d}=5 \times 10^{7} \mathrm{yr}$. Instead, four new groups of pulsars appear, which we denote $\mathrm{a}_{1}, \mathrm{~b}_{1}, \mathrm{~h}_{1}$, and $\mathrm{K}$.

Radio pulsars of group $\mathrm{a}_{1}$ are produced by binaries with $M_{1} \simeq 13 M_{\odot}, M_{2} \simeq 2-3 M_{\odot}$, and $a \simeq(1-2) \times$ $10^{3} R_{\odot}$. These pulsars undergo a superaccretion stage only, during which they accumulate a mass $\Delta M \simeq$ $0.01-0.2 M_{\odot}$. At the same time, pulsars of the adjacent group A (Fig. 5b) increase their masses only during an accretion stage.

Radio pulsars of group $b_{1}$ are produced by binaries with $M_{1} \simeq 17 M_{\odot}, M_{2} \simeq 2-3 M_{\odot}$, and $a \simeq(5-7) \times$ $10^{2} R_{\odot}$. These pulsars increase their masses purely due to superaccretion $\left(\Delta M \simeq 0.5-0.7 M_{\odot}\right)$.

Radio pulsars of group $h_{1}$ are produced by binaries with $M_{1} \simeq 25 M_{\odot}, M_{2} \simeq 4-5 M_{\odot}$, and $a \simeq$ $10^{3} R_{\odot}$. These pulsars accumulate mass in superaccretion $\left(\Delta M \simeq 0.03 M_{\odot}\right)$ and hyperaccretion $(\Delta M \simeq$ $\left.0.6 M_{\odot}\right)$ stages.

Radio pulsars of group $\mathrm{K}$ also undergo superaccretion and hyperaccretion phases, during which their masses increase by $\Delta M \simeq 0.1 M_{\odot}$ and up to $\Delta M \simeq$ $1 M_{\odot}$, respectively. Such a large mass increase in the hyperaccretion stage is possible thanks to the small initial separation of the components, $a \simeq 10^{2} R_{\odot}$. The initial component masses for this group are close to those for group $\mathrm{h}_{1}: M_{1} \simeq 25 M_{\odot}$ and $M_{2} \simeq 5 M_{\odot}$.

The radio pulsars of groups A, C, B, I, F, H, and G in Fig. 5b differ from their counterparts in Fig. 5a only in the strength of their magnetic fields, which have changed with $t_{d}$ in accordance with (9). The accretion stages, their chronology, and the values of $\Delta M \simeq 1 M_{\odot}$ remain the same. We note only that the pulsars of group $\mathrm{F}$ in Fig. $5 \mathrm{~b}$ accumulate $\Delta M \simeq$ $0.2 M_{\odot}$ in the superaccretion stage, in contrast to the corresponding pulsars in Fig. 5a, which accumulate only $\Delta M \simeq 0.01 M_{\odot}$.

\section{Magnetic-field Decay Time Scale $10^{7}$ yr}

Let us now compare the groups of radio pulsars in Figs. $5 \mathrm{~b}$ and $5 \mathrm{c}$. Groups $\mathrm{a}_{1}, \mathrm{a}_{2}, \mathrm{~h}_{1}, \mathrm{I}, \mathrm{H}, \mathrm{K}$, and $\mathrm{G}$ have disappeared, while the qualitatively new groups $\mathrm{f}_{1}$ and $\mathrm{f}_{2}$ have emerged.

Radio pulsars of group $\mathrm{f}_{1}$ (Fig. $5 \mathrm{c}$ ) are produced by binaries with $M_{1} \simeq 10 M_{\odot}, M_{2} \simeq 10 M_{\odot}$, and $a \simeq$ $10^{2} R_{\odot}$. These pulsars undergo a superaccretion stage only, accumulating a mass $\Delta M \simeq 0.3 M_{\odot}$.

Like the group-F pulsars, radio pulsars of group $\mathrm{f}_{2}$ accumulate mass during superaccretion $(\Delta M \simeq$ $\left.0.01-0.1 M_{\odot}\right)$ and hyperaccretion $(\Delta M \simeq$ $\left.0.5-1 M_{\odot}\right)$ stages. However, in constrast to the group- $F$ pulsars, the magnetic-field strength decays to its minimum value, due to the fact that the optical donors are less massive $\left(M_{2} \simeq 3 M_{\odot}\right)$ than the donors of the group-F pulsars $\left(M_{2} \simeq 5-8 M_{\odot}\right)$. For this reason, the nuclear time scales for the donors in $\mathrm{f}_{2}$ group are long enough for the pulsar magnetic fields to decay. The initial masses of the primaries of the binaries giving rise to group $\mathrm{f}_{2}$ radio pulsars are $M_{1} \simeq 12-14 M_{\odot}$, while the initial semimajor orbital axes are $a \simeq(4-5) \times 10^{2} R_{\odot}$.

The remaining groups of radio pulsars in Fig. $5 \mathrm{c}$, i.e., A, B, C, and F, are qualitatively the same as the corresponding groups in Figs. 5a and 5b. The only difference between the two analogous groups of radio pulsars is that the magnetic-field strength changes in accordance with (9), with $t_{d}=10^{7} \mathrm{yr}$.

Thus, the model computations taking into account the common-envelope (hyperaccretion) stage indicate that the masses of radio pulsars in binaries with white dwarfs are in the range $m_{\mathrm{PSR}} \simeq 1.35-2.5 M_{\odot}$. Such considerable growths in the masses of radio pulsars can come about due to both hyperaccretion and ordinary accretion from a low-mass optical donor. Note that the radio pulsars can sometimes increase their masses to the Oppenheimer-Volkoff limit, $m_{\mathrm{OV}} \simeq 2.5 M_{\odot}$. 

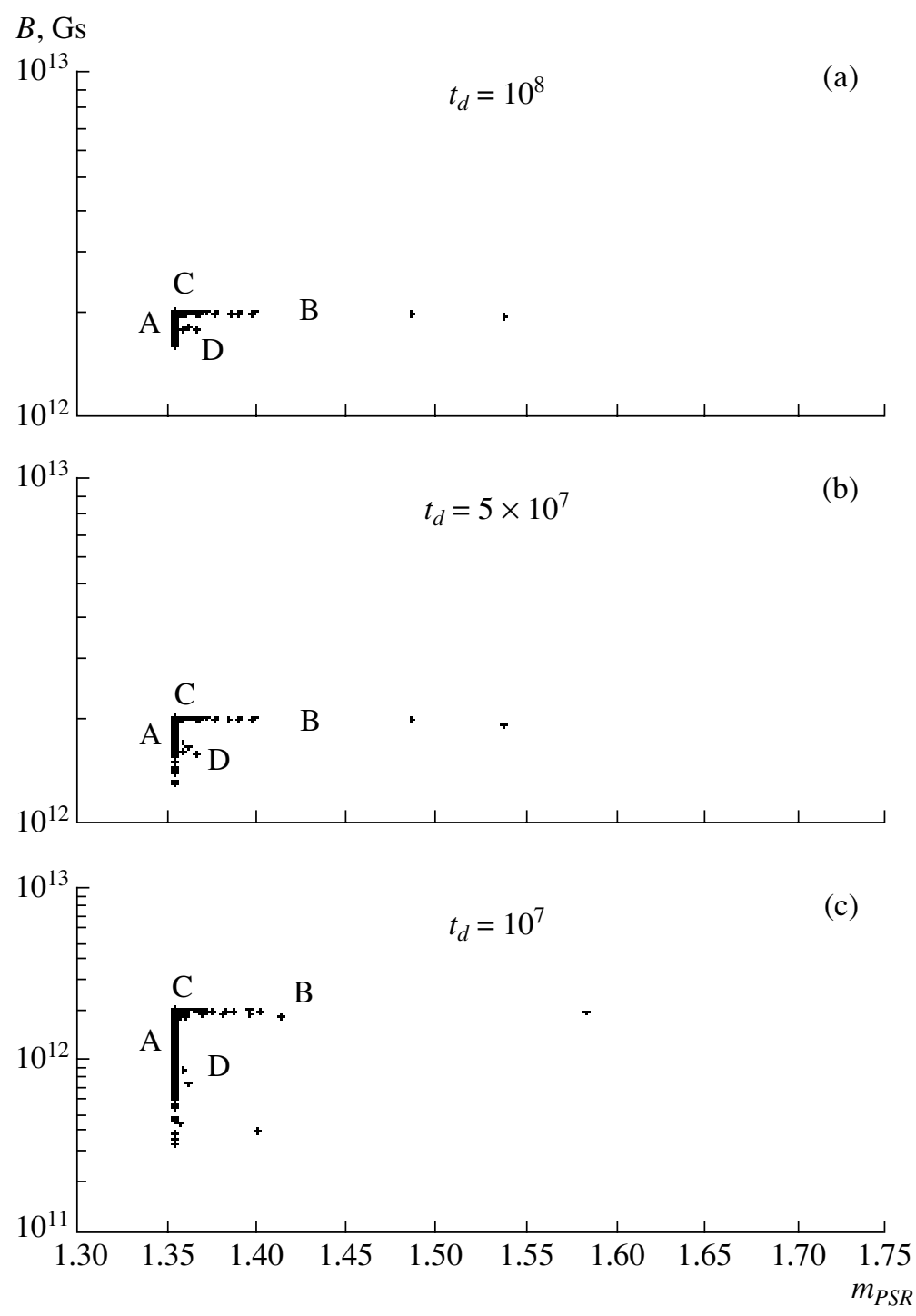

Fig. 6. Same as Fig. 4, but without a hyperaccretion stage.

\section{Radio Pulsars in PSR $+N S$ Binaries (Population Synthesis without Hyperaccretion)}

The hyperaccretion stage is not well understood. Neither the time scale, nor the rate of infall of matter onto the surface of the neutron star during this stage are known. For this reason, we carried out an additional set of population-synthesis computations without including hyperaccretion; i.e., in which the radio pulsars could increase their masses only via conventional accretion and superaccretion.

As in the previous case, we carried out the population-synthesis computations for 19.5 million initial stellar pairs, with the same ranges of the initial stellar parameters. These computations produced $\sim 7 \times 10^{4} \mathrm{PSR}+\mathrm{NS}$ binaries. The masses $m_{\mathrm{PSR}}$ and magnetic-field strengths $B$ of the radio pulsars for magnetic-field decay time scales of $t_{d}=10^{8}, 5 \times 10^{7}$, and $10^{7} \mathrm{yr}$ are shown in Figs. $6 \mathrm{a}-\mathrm{c}$, respectively. We can see that the locations of the groups of radio pulsars are similar to those for the other computations. Varying $t_{d}$ resulted in quantitative changes only, namely, in a reduction of the magnetic-field strength with decreasing $t_{d}$.

Let us see how the population-synthesis computations without a common-envelope stage (Fig. 6) affected the locations of groups A, B, C, and D (Fig. 4). A comparison of Figs. 4 and 6 shows that the masses of the radio pulsars in groups B, C, and D have decreased by $\Delta M \simeq 0.5 M_{\odot}$ : the positions of these 

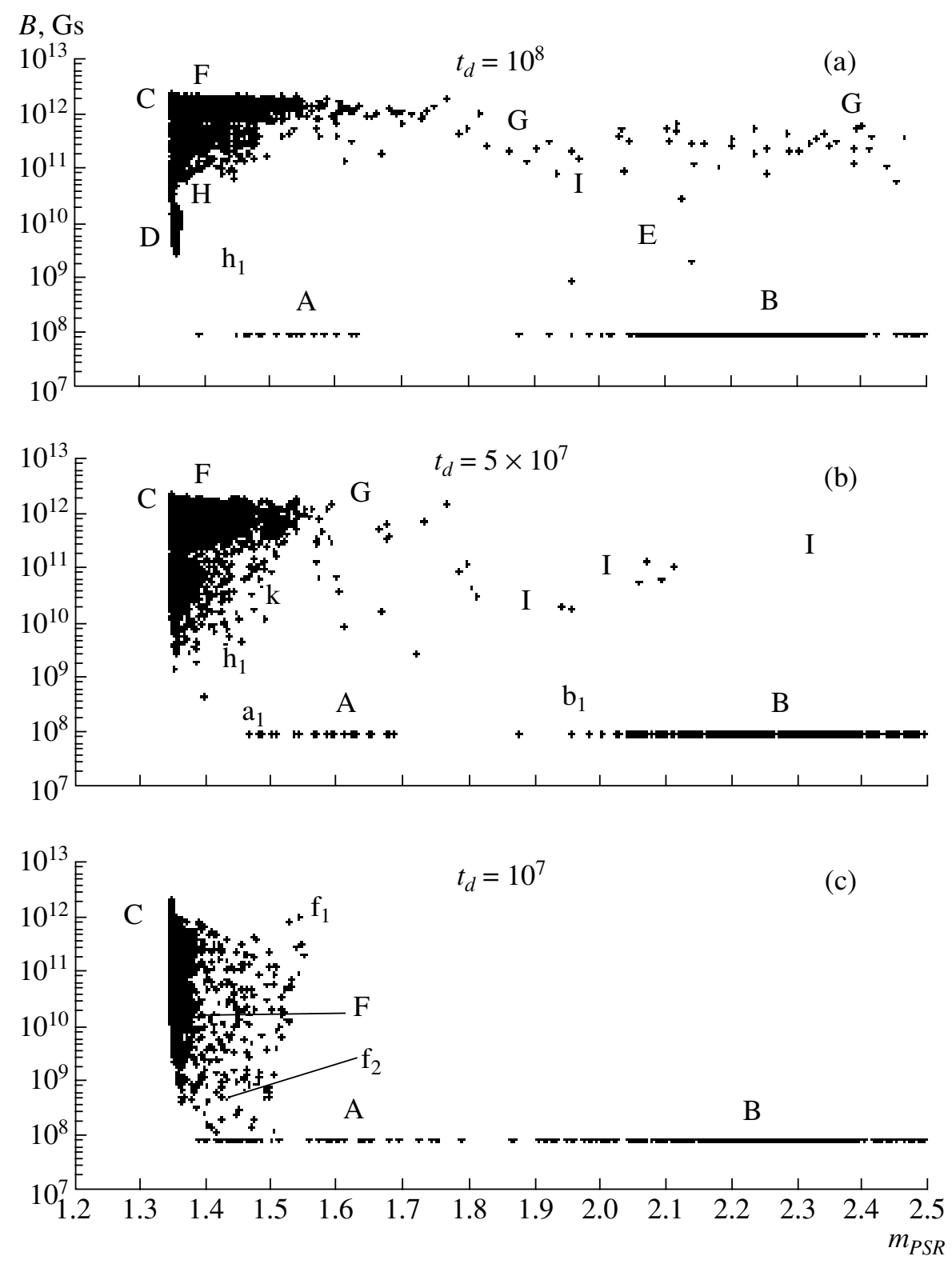

Fig. 7. Same as Fig. 4 for PSR+WD systems $\left(\sim 16 \times 10^{4}\right.$ objects $)$ for the computations without hyperaccretion.

pulsars are shifted along the horizontal axis toward lower masses. The positions of the group-A radio pulsars did not change, since these pulsars do not experience accretion, superaccretion, hyperaccretion stages during their evolution. The initial parameters of the binaries giving rise to the pulsars in groups A, B, C, and D in Fig. 6 are identical to the corresponding parameters for these groups of pulsars in Fig. 4, listed above.

The radio pulsars of groups B, C, and D increase their masses only in a superaccretion stage. Figure 6 shows that the mass accumulated in this stage can range from $\Delta M \simeq 0.01$ to $\Delta M \simeq 0.20 M_{\odot}$. The accumulation of matter occurs over $t \lesssim t_{d}$.

\section{Radio Pulsars in PSR+WD Binaries (Population} Synthesis without a Common-envelope Stage)

The population-synthesis computations for the 19.5 million initial binaries carried out without a common-envelope (hyperaccretion) stage produced $\sim 16 \times 10^{4}$ PSR+WD systems. The masses and magnetic-field strengths for these pulsars are shown in Figs. $7 \mathrm{a}-\mathrm{c}$ for magnetic-field decay time scales of $t_{d}=10^{8}, 5 \times 10^{7}$, and $10^{7} \mathrm{yr}$, respectively.

Let us compare the positions of the groups of radio pulsars in Figs. 7 and 5. In Fig. 7a, groups F and H, whose pulsars accumulate mass predominantly via hyperaccretion, are shifted horizontally toward lower masses compared to those for the same groups in 

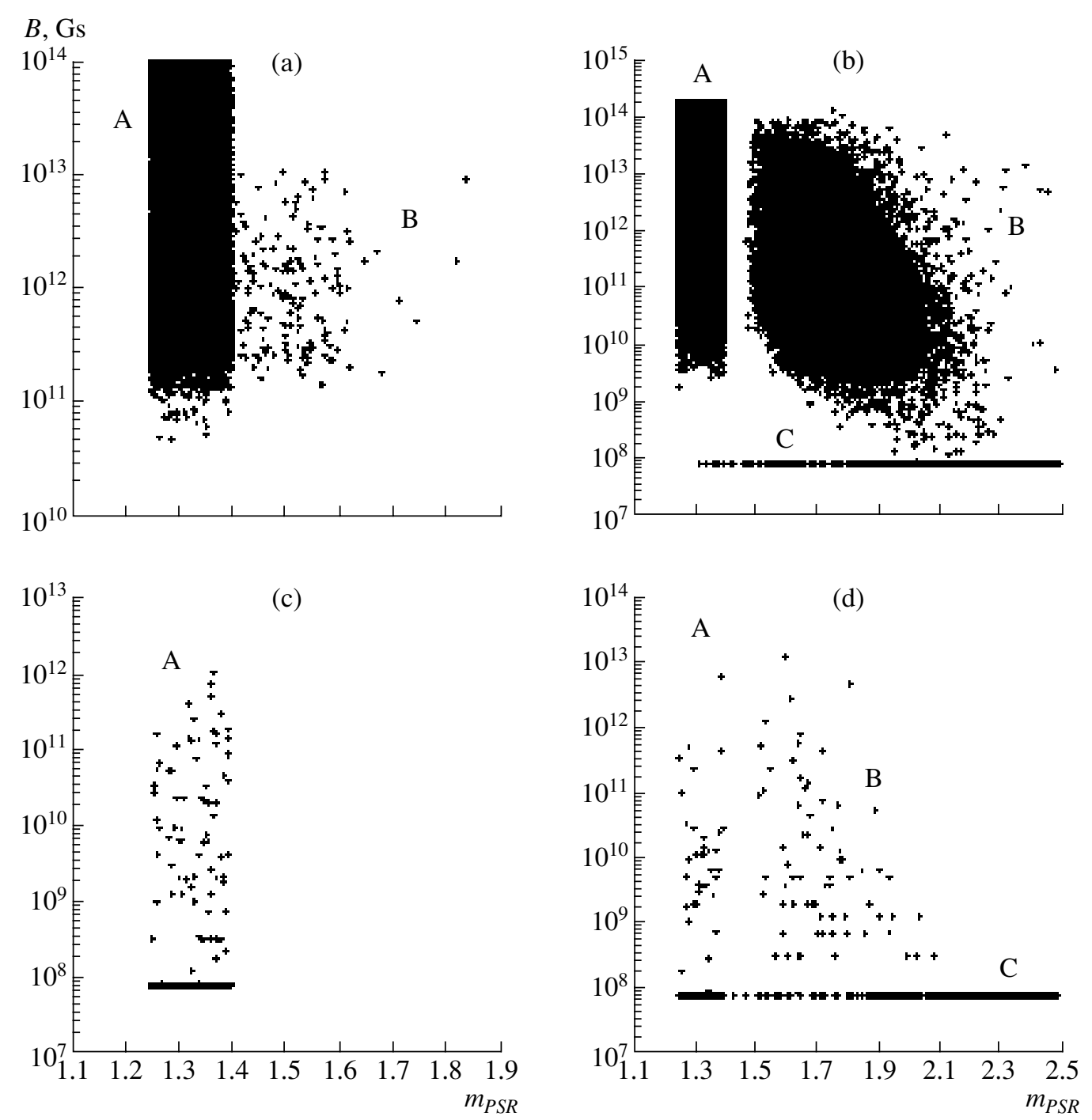

Fig. 8. (a) Masses and magnetic-field strengths of radio pulsars in PSR+NS pairs $\left(\sim 24 \times 10^{4}\right.$ systems $)$ obtained in the population-synthesis computations for 72 million initial binaries taking into account a hyperaccretion stage and allowing for spreads in the initial masses and magnetic-field strengths of the radio pulsars in the ranges $m_{\mathrm{PSR}}=1.25-1.40 M_{\odot}$ and $B=2 \times 10^{11}-2 \times 10^{14}$ G. (b) Same as (a) for radio pulsars in PSR+WD pairs $\left(\sim 43 \times 10^{4}\right.$ systems). (c) Masses and magnetic-field strengths of radio pulsars in PSR +NS pairs after correcting the observed number of such systems for selection effects ( $\sim 900$ systems). (d) Same as (c) for radio pulsars in PSR+WD binaries ( $\sim 600$ systems).

Fig. 5a. In Fig. $7 \mathrm{~b}$, groups $\mathrm{F}, \mathrm{K}$, and $\mathrm{h}_{1}$ are likewise shifted toward lower masses compared to those for these groups in Fig. 5b. The same occurs for groups F and $\mathrm{f}_{1}$ in Fig. $7 \mathrm{c}$ (compare to Fig. $5 \mathrm{c}$ ). The radio pulsars in the remaining groups, which accumulate mass in accretion and superaccretion stages, remained in the same positions. The initial binary parameters for the various groups of radio pulsars represented in Fig. 7 are listed above.

Note that a radio pulsar can increase its mass to the Oppenheimer-Volkoff limit, $m_{\mathrm{OV}} \simeq 2.5 M_{\odot}$, without passing through a common-envelope stage. It is possible to increase the radio pulsar's mass to $m_{\mathrm{PSR}} \simeq 2.5 M_{\odot}$ only if the stage of accretion from the low-mass donor is long (for radio pulsars of groups B and I in Fig. 7).

Radio Pulsars in PSR+NS and PSR+WD Binaries (Population Synthesis Allowing Ranges in the Initial Binary Parameters and All Types of Accretion)

In the population-synthesis algorithm described above, the initial magnetic-field strength and initial pulsar mass were set equal to $B=2 \times 10^{12} \mathrm{G}$ and $m_{\mathrm{PSR}}=1.35 M_{\odot}$. Since these initial values can vary, we carried out an additional population-synthesis run for 72 million initial binaries allowing initial 
magnetic-field strengths from $B=2 \times 10^{11} \mathrm{G}$ to $B=2 \times 10^{14} \mathrm{G}$ and initial pulsar masses in the range $m_{\mathrm{PSR}}=1.25-1.40 M_{\odot}$. The range of initial pulsar masses was based on the masses of the neutron star in the B1913+16 binary system and of the radio pulsar in the J0737-3039 binary. In the B1913+16 system (the Hulse-Taylor pulsar, PSR+NS) the mass of the neutron star, which is definitely not accreting matter from the optical donor, is $m_{N S}=$ $1.3873 \pm 0.0006 M_{\odot}$ [13]. The mass of the pulsar in the J0737-3039 system (PSR+PSR), which likewise does not accrete from an optical companion is $m_{\mathrm{PSR}}=1.250 \pm 0.010 M_{\odot}[20]$.

The parameters of the PSR+NS $\left(\sim 24 \times 10^{4}\right)$ and PSR+WD $\left(\sim 43 \times 10^{4}\right)$ systems obtained via the population synthesis are represented in Figs. 8a and $8 \mathrm{~b}$. Group $-\mathrm{A}$ radio pulsars in PSR $+\mathrm{NS}$ binaries (Fig. 8a) do not increase their masses during their evolution. The range of masses of the group $\mathrm{A}$ pulsars is determined purely by the range of initial pulsar masses. The masses of group-B radio pulsars (Fig. 8b) grew predominantly due to hyperaccretion $\left(\Delta M>0.1 M_{\odot}\right)$ and, to a substantially lesser degree, superaccretion $\left(\Delta M \simeq 0.01-0.1 M_{\odot}\right)$.

The group-A radio pulsars in PSR + WD binaries (Fig. 8b) did not undergo accretion, superaccretion, or hyperaccretion stages. The range of their masses is determined purely by the range of the initial masses. The group-B radio pulsars (Fig. 8b) accumulated masses of $\Delta M \simeq 0.2-1.2 M_{\odot}$ via accretion, superaccretion, and hyperaccretion from optical companions with masses $M_{2} \simeq 5-10 M_{\odot}$. The group-C radio pulsars (Fig. 8b) accumulated masses of $\Delta M \simeq$ $0.1-1.2 M_{\odot}$ via accretion from their low-mass $\left(M_{2} \simeq\right.$ $1-3 M_{\odot}$ ) optical companions.

The lower panels of Fig. 8 (c, d) show the samples of PSR+NS and PSR+WD binaries that remain after allowing for observational selection effects. The selection was based on the lifetimes of PSR $+\mathrm{NS}$ and $\mathrm{PSR}+\mathrm{WD}$ binaries. After this effect was taken into account, there remained $\sim 900 \mathrm{PSR}+\mathrm{NS}$ systems (Fig. 8c) and $\sim 600$ PSR+WD systems (Fig. 8d).

The results of the population syntheses corrected for observational selection effects indicate that the masses of radio pulsars in observed PSR+NS systems are confined to the range of their initial masses: from $1.25 M_{\odot}$ to $1.44 M_{\odot}$ (Fig. 8d). Correcting for observational selection effects causes pulsars that accumulate mass via hyperaccretion to disappear (Figs. 8a, 8c). All previously selected groups of pulsars in PSR+WD binaries survived after correcting for observational selection effects (Figs. 8b and $8 d$ ), though the number of objects in each group decreased. The population-synthesis results indicate that observed masses of radio pulsars in binaries with white dwarfs should be in the range $m_{\mathrm{PSR}}=$ $1.25 M_{\odot}-2.5 M_{\odot}$.

\section{CONCLUSION}

The population-synthesis results are in qualitative and quantitative agreement with the observed parameters of radio pulsars in binaries with degenerate companions (Figs. 2, 3, and 8).

Our population syntheses show that PSR+NS systems can contain radio pulsars with masses exceeding the Chandrasekhar limit $\left(M_{C h} \simeq 1.4 M_{\odot}\right)$. The masses of radio pulsars in PSR $+\mathrm{NS}$ binaries grow predominantly via hyperaccretion of matter from a massive donor $\left(M_{2}>10-15 M_{\odot}\right)$. However, it is difficult to observe massive radio pulsars in PSR $+\mathrm{NS}$ systems (Fig. 8c), since the lifetimes of PSR+NS pairs with "heavy" radio pulsars $\left(m_{\mathrm{PSR}}>1.4 M_{\odot}\right)$ are fairly short. The model computations suggest that radio pulsars with masses $m_{\mathrm{PSR}}$ considerably higher than the Chandrasekhar limit, $M_{C h} \simeq 1.4 M_{\odot}$, are likely to be present among PSR $+\mathrm{WD}$ binaries (Fig. 8c).

The mechanisms via which radio pulsars in PSR+WD pairs accumulate mass are more diverse. In the course of the system's evolution, the pulsar mass can increase due to hyperaccretion, superaccretion, and accretion. Radio pulsars in PSR+WD systems gain the most mass due to accretion of matter from their low-mass optical companions $\left(M_{2}=1-3 M_{\odot}\right)$. The population-synthesis results indicate that these radio pulsars have weak magnetic fields, $B \simeq 10^{8} \mathrm{G}$ (group $\mathrm{C}$ in Figs. $8 \mathrm{c}$ and $8 \mathrm{~d}$ ), because their low-mass optical companions fill their Roche lobes on the nuclear time scale, $t \simeq 10^{9} \mathrm{yr}$. The magnetic fields of the radio pulsars have decayed by two to three orders of magnitude by the onset of the accretion. The radio pulsars $\mathrm{J} 1012+5307$ and J1713+0747 (Table 2, Figs. 2 and 3) may belong to this group. The accreted mass supplied by lowmass optical donors is able to increase the mass of the radio pulsar to the Oppenheimer-Volkoff limit, $M_{\mathrm{OV}} \simeq 2.5 M_{\odot}$.

Based on the population-synthesis results, we expect the observed masses of radio pulsars in $\mathrm{PSR}+\mathrm{NS}$ and PSR+WD binaries to display a significant scatter, from $1.3 M_{\odot}$ to $M_{\mathrm{OV}}$, due to the accumulation of matter from optical companions. The observed central masses of radio pulsars (Tables 1 and 2 and Fig. 3) are consistent with the populationsynthesis results. 


\section{ACKNOWLEDGMENTS}

The authors are grateful to A.G. Kuranov, K.A. Postnov, and M.E. Prokhorov for valuable remarks and advice. This study was supported by the Russian Foundation for Basic Research (project no. 02-02-17524) and the program "Leading Scientific Schools of Russia” (NSh 388.2003.2).

\section{REFERENCES}

1. A. M. Cherepashchuk, Usp. Fiz. Nauk 166, 809 (1996) [Phys.-Usp. 39, 759 (1996)].

2. A. M. Cherepashchuk, Usp. Fiz. Nauk 173, 345 (2003) [Phys.-Usp. 46, 335 (2003)].

3. Ya. B. Zel'dovich, Dokl. Akad. Nauk SSSR 155, 67 (1964) [Sov. Phys. Dokl. 9, 195 (1964)].

4. E. E. Salpeter, Astrophys. J. 140, 796 (1964).

5. N. I. Shakura and R. A. Sunyaev, Astron. Astrophys. 24, 337 (1973).

6. J. E. Pringle and M. J. Rees, Astron. Astrophys. 21, 1 (1972).

7. I. D. Novikov and K. S. Thorne, in Black Holes, Ed. by C. De Witt and B. S. De Witt (Gordon and Breach, London, 1973), p. 343.

8. R. A. Chevalier, Astrophys. J. 411, L.33 (1993).

9. V. M. Lipunov, K. A. Postnov, and M. E. Prokhorov, The Scenario Machine: Binary Star Population Synthesis, Ed. by R. A. Sunyaev (Astrophysics and Space Physics Reviews, Harwood Academic Publishers, 1996), Vol. 9.

10. V. M. Lipunov, Astron. Zh. 59, 888 (1982) [Astron. Rep. 26, 537 (1982)]

11. Ya. B. Zel'dovich, L. N. Ivanova, and D. K. Nadezhin, Astron. Zh. 49, 253 (1972) [Ästron. Rep. 16, 209 (1972)].

12. M. M. Basko and R. A. Sunyaev, Astron. Astrophys. 42, 311 (1975).

13. S. E. Thorsett and D. Chakrabarty, Astrophys. J. 512, 288 (1999)

14. D. J. Nice, R. W. Sayer, and J. H. Taylor, Astrophys. J. 466, L.87 (1996).

15. M. Konacki, A. Wolszczan, and I. H. Stairs, Astrophys. J. 589, 495 (2003).

16. I. H. Stairs, S. E. Thorsett, J. H. Taylor, and A. Wolszczan, Astrophys. J. 581, 501 (2002).

17. J. M. Weisberg and J. H. Taylor, astro-ph/0211217.

18. N. Wex, V. Kalogera, and M. Kramer, Astrophys. J. $528,401(2000)$

19. Z. Arzoumanian, M. Cordes, and I. Wasserman, Astrophys. J. 520, 696 (1999).

20. A. G. Lyne, M. Burgay, M. Kramer, et al., astro$\mathrm{ph} / 0401086$.

21. W. Straten, M. Bailes, M. C. Britton, et al., Nature 412,158
22. E. M. Splaver, D. J. Nice, Z. Arzoumanian, et al., Astrophys. J. 581, 509 (2002).

23. F. Camilo, D. J. Nice, J. A. Shrauner, and J. H. Taylor, Astrophys. J. 469, L819 (1996).

24. M. Bailes, S. M. Ord, H. S. Knight, and A. W. Hotan, astro-ph/0307468.

25. V. M. Kaspi, A. G. Lyne, R. N. Manchester, et al., Astrophys. J. 543, 321 (2000).

26. D. J. Nice, E. M. Splaver, and I. H. Stairs, in $I A U$ Symp. No. 218: Young Neutron Stars and Their Environments, Ed. by F. Camilo and B. M. Gaensler (Astron. Soc. Pacif., San Francisco, 2004), p. 49; astro-ph/0311296.

27. D. J. Nice, E. M. Splaver, and I. H. Stairs, in $R a-$ dio Pulsars, Ed. by M. Bailes, D. J. Nice, and S. E. Thorsett, Astron. Soc. Pacif. Conf. Ser., vol. 302 , in press; astro-ph/0210637.

28. M. H. van Kerkwijk and S. R. Kulkarni, Astrophys. J. 516, L25 (1999).

29. J. Kaluzny, S. M. Rucinski, and I. B. Thompson, Astron. J. 125, 1546 (2003).

30. N. D'Amico, A. G. Lyne, R. N. Manchester, A. Possenta, and F. Camilo, Astrophys. J. 548, L171 (2001).

31. N. D'Amico, A. Possenti, R. N. Manchester, et al., Astrophys. J. 561, L89 (2001).

32. W. Coburn, W. A. Heindl, R. E. Rothschild, et al., Astrophys. J. 580, 394 (2002).

33. P. G. Jonker, M. van der Klis, and P. J. Groot, Mon. Not. R. Astron. Soc. 339, 663 (2003).

34. P. G. Jonker and M. van der Klis, Astrophys. J. 553, L43 (2001).

35. A. P. Reynolds, H. Quaintrell, M. D. Still, et al., Mon. Not. R. Astron. Soc. 288, 43 (1997).

36. H. Tananbaum, H. Gursky, E. Kellogg, et al., Astrophys. J. 174, L143 (1972).

37. M. K. Abubekerov, E. A. Antokhina, and A. M. Cherepashchuk, Astron. Zh. 81, 108 (2004) [Astron. Rep. 48, 89 (2004)].

38. R. Giacconi, H. Gursky, E. Kellogg, et al., Astrophys. J. 167, L67 (1971).

39. R. L. Kelley, J. G. Jernigan, A. Levine, et al., IAU Circ. No. 3632(2) (1981).

40. A. La Barbera, L. Burderi, T. Di Salvo, et al., Astrophys. J. 553, 375 (2001).

41. P. Kahabka and X.-D. Li, Astron. Astrophys. 345, 117 (1999).

42. N. R. Robba, L. Burdert, T. Di Salvo, et al., Astrophys. J. 562, 950 (2001).

43. J. E. McClintock, S. Rappaport, P. C. Joss, et al., Astrophys. J. 206, 99 (1976).

44. V. M. Lipunov, K. A. Postnov, and M. E. Prokhorov, Mon. Not. R. Astron. Soc. 288, 245 (1997).

Translated by L. Yungel'son 